\title{
Potencialidades e fragilidades da educação a distância no contexto da Atenção Básica: estudo exploratório qualitativo
}

\author{
Natalia Conteçote Russoํㅜ, Karina Rubia Nunes², Carla Maria Vieira³, Jéssica Emy \\ Komuro $^{4}$ e Maria Rita Marques de Oliveira ${ }^{5}$
}

A educação a distância como estratégia para o incremento das ações de alimentação e nutrição na atenção primária no Sistema único de Saúde/SUS é o tema central desse artigo, com o objetivo de analisar uma experiência de educação a distância para a promoção da segurança alimentar e nutricional no contexto da atenção básica. Os dados foram gerados a partir da interação dos educandos e tutores, em ambiente virtual. A partir do referencial de análise de conteúdo, os resultados foram organizados em categorias e discutidos à luz do arcabouço teórico da Saúde Coletiva e da Educação Alimentar e Nutricional, resultando em três categorias: O processo educacional e o educando em ambiente virtual; $O$ papel do tutor na modalidade de EAD: Ações intersetoriais e territorialização. Concluiu-se que a educação a distância é uma tecnologia que promove mudanças, quando na perspectiva da aproximação da teoria e prática, da ressignificação do processo de trabalho e do empoderamento do educando para ações de transformação na atenção básica.

Palavras-chave: Educação a Distância, Atenção Primária à Saúde, Segurança Alimentar e Nutricional.

\section{Potentialities and fragilities of distance learning in the context of Primary Care: qualitative exploratory study}

The distance education as a strategy to increase food and nutrition actions in primary health care in the Unified Health System/Brazil (SUS) is the central theme of this article, with the objective of evaluating the process of distance education for the promotion of food and nutritional security in the context of primary care. The data were generated from the interaction between the students and tutors, in on-line environment and categorized

${ }^{1}$ Departamento de Enfermagem - Faculdade de Medicina de Botucatu. Universidade Estadual Paulista Júlio de Mesquita Filho (UNESP). ORCID - https://orcid.org/00000001-5064-8778.

2 Pós-Doutoranda. Departamento de Economia, Administração e Educação - Faculdade de Ciências Agrárias e Veterinárias de Jaboticabal. Centro de Ciência e Tecnologia e inovação para Soberania e Segurança Alimentar e Nutricional da INTERSSAN - UNESP. ORCID - https://orcid.org/0000-0002-2888-4763. Endereço para correspondência: Rua Ricardo Alpídio Sarzi no 467 - Jardim Ouro Verde - Botucatu - SP/Brasil. E-mail: karina.rubia@unesp.br

${ }^{3}$ Pós-Doutoranda. Departamento de Enfermagem - Faculdade de Medicina de Botucatu. Centro de Ciência e Tecnologia e Inovação para Soberania e Segurança Alimentar e Nutricional da INTERSSAN - UNESP. ORCID - https://orcid.org/0000-0002-4486-4527.

${ }^{4}$ Mestre - Departamento de Enfermagem - Faculdade de Medicina de Botucatu - UNESP. ORCID - https://orcid.org/0000-0003-2854-0685.

${ }^{5}$ Professora Assistente Doutora - Departamento de Educação - Instituto de Biociências de Botucatu (IBB). Centro de Ciência e Tecnologia e Inovação para Soberania e

Segurança Alimentar e Nutricional da INTERSSAN - UNESP. ORCID - https://orcid.org/0000-0003-1226-4364. 
from the content analysis framework. The discussion of the results was supported by the theoretical framework of Public Health and Nutrition Education. The categories of analysis are "The educational process and the student in a virtual environment", "The role of the tutor in the EAD modality" and "Theintersectoral actions and territorializing". It was concluded that distance education is a technology that promotes change, when used to approximate theory and practice, to re-signify work and to empower the learner for transformative actions in primary care.

Keywords: Distance Learning, Primary Health Care, Food Policy.

\section{INTRODUÇÃO}

A educação a distância, enquanto modalidade de ensino e aprendizagem ofertada para aqueles que já estão inseridos em campos de atuação profissional, vem sendo reconhecida na literatura ${ }^{[1]}$ como uma importante contribuição para a formação e qualificação profissional. Processos educacionais desenvolvidos em ambientes virtuais são apontados, por diferentes autores ${ }^{[2]}$ com potencialidade de estabelecer parcerias, redes de apoio e para aproximar os saberes e práticas, que se reinventam a cada dia, a partir das demandas reais e necessidades que emergem na dinâmica de relações sociais, em número ampliado de comunidades e territórios.

Profissionais que atuam na Atenção Básica (AB) têm contato diário com essas dinâmicas sociais e, portanto, desenvolvem ao longo de sua atuação profissional a capacidade de leitura da realidade. Contudo, sua capacidade de reflexão e de mediação demandam aprimoramento contínuo e permanente em todos os campos de conhecimento, tendo em vista a complexidade e multiplicidade de fatores sociais, culturais e econômicos, determinantes dos problemas e situações de vulnerabilidade.

Avanços significativos no que diz respeito à organização de referências institucionais para o desenvolvimento das ações de alimentação e nutrição na $A B[3]$ têm sido verificados nos documentos oficiais do Ministério da Saúde. Contudo, muitas lacunas são apontadas na literatura e o enfrentamento da obesidade e de problemas de saúde vinculados ao excesso de peso, a exemplo das doenças crônicas não transmissíveis, exige ampliação da atuação de nutricionistas na $\mathrm{AB}$ e também maior oferta de cursos para qualificar ainda mais o desenvolvimento de ações de alimentação e nutrição e de projetos de promoção de segurança e soberania alimentar nas comunidades.

A oferta de cursos na modalidade de EAD é uma alternativa interessante nesse contexto pois aumenta a possibilidade de acesso que demandam, por sua vez, processos avaliativos contínuos e reflexão teórica, visto que envolvem relações entre educadores e educandos, questões metodológicas, produtos educacionais, entre outros elementos do processo de ensino. Nesse contexto, este artigo tem como objetivo analisar uma experiência de um curso de EAD ofertado pela Rede-SANS, estruturado para atender necessidades de equipes de $\mathrm{AB}$, com foco na promoção da Segurança Alimentar e Nutricional em territórios, em situação de vulnerabilidade.

\section{METODOLOGIA}

A Rede de Municípios Promotores da Segurança Alimentar e Nutricional Sustentável e Solidária (Rede-SANS) ${ }^{[4]}$, consolidada durante a execução de convênio entre o Ministério da Ciência, Tecnologia e Inovação - MCTI e a Financiadora de Estudos e Projetos -FINEP/Conselho Nacional de Desenvolvimento Científico e Tecnológico- $\mathrm{CNPq}$, realizou diagnóstico qualitativo e quantitativo das ações de alimentação e nutrição na atenção primária do SUS, em 65 municípios do estado de São Paulo. Desse convênio emergiram municípios com realidades de maior vulnerabilidade para as ações de alimentação e nutrição, bem como, serviços e profissionais com necessidade de formação para a qualificação do trabalho na $\mathrm{AB}$ do SUS.

A elaboração do projeto Rede-SANS, foi guiada pelo rigor do método científico, porém sem desconsiderar a dinâmica da prática social. A relação dialógica com os municípios, o trabalho orientado pela 
problematização, o conceito de rede e a autonomia de cada parte em um contexto de necessidades comuns, conformaram a base teórica de trabalho do grupo de profissionais ${ }^{[4]}$. Além disso, a fundamentação teórica no campo da segurança alimentar, da nutrição em saúde coletiva e da educação alimentar e nutricional (EAN), também guiaram a construção de ações da equipe.

O curso de extensão a distância nominado Interdisciplinaridade, Alimentação e Nutrição (Interanutri) - Modalidade Agente, contexto desta pesquisa, foi ofertado pela Rede-SANS/UNESP, a partir da demanda de formação para a qualificação do trabalho na atenção básica. O curso foi destinado a profissionais da Atenção Básica, de municípios que aderiram a Rede-SANS[4]. A adesão à Rede-SANS ocorreu mediante a oficialização de um documento assinado pelo poder público municipal que formalizou o vínculo mediado pelo Instituto de Biociências de Botucatu-IBB. A contrapartida dos municípios que participaram foi a indicação de profissionais para atuarem como tutores no curso EAD.

O curso foi realizado no ano de 2012, com o objetivo de formação de profissionais para o desenvolvimento de ações de promoção da alimentação saudável, adequada e solidária com envolvimento da comunidade.

Foi utilizado o Modular Object Oriented Distance Learning (Moodle), conjunto de ferramentas computacionais (fórum, chat, tarefa com envio de arquivo e lição) que apoiaram a aprendizagem e execução de um curso a distância. O Moodle é um software gratuito, de livre acesso e utilizado como ferramenta de um processo dinâmico de aprendizagem por meio de trocas $[$ [].

O curso foi organizado em 15 módulos com total de 60 horas. Os módulos foram estruturados com discussão em fórum, elaboração de narrativas, leituras e sínteses reflexivas. Foi disponibilizada uma biblioteca com vídeo aula e materiais bibliográficos de apoio para o desenvolvimento das atividades de ensino-aprendizagem e do projeto de intervenção que os educandos planejaram e executaram com suas comunidades. O curso contou com dois momentos presenciais para cadastro e familiarização com a plataforma Moodle e um para apresentação dos projetos de intervenção.
A equipe que organizou a proposta do curso e todo seu conteúdo, se reuniu com frequência quinzenal para discussão e sistematização do material a ser disponibilizado para os alunos na plataforma. A equipe era composta por uma coordenadora das atividades do curso, uma nutricionista, uma enfermeira, um psicólogo, uma educadora física, uma agrônoma e um técnico responsável pela postagem dos conteúdos na plataforma Moodle. Os temas abordados durante o curso envolveram a segurança alimentar e nutricional no contexto comunitário, a intersetorialidade como estratégia, o conceito ampliado de saúde, a educação como princípio de promoção da pessoa e acesso à cidadania, o Direito Humano à Alimentação Adequada e consumo e meio ambiente. Essa proposta teórica promoveu a oportunidade dos educandos ampliarem leitura de realidade por meio da aproximação com o referencias teóricos da SAN.

O projeto de intervenção prática realizado com a comunidade foi construído pelos educandos como um projeto com estrutura acadêmica: justificativa, definição do objetivo e da metodologia participativa, com estratégias para envolvimento de moradores da comunidade. Todos os projetos foram orientados a ter como referência o campo de saber da Educação em Saúde e alinhados a Política Nacional de Segurança Alimentar e Nutricional (PNSAN) vigente na época.

Para a reflexão crítica dessa experiência de curso EAD os dados foram obtidos em fontes secundárias, extraídos em ambiente virtual de aprendizagem (AVA), configurando-se como um estudo exploratório qualitativo. Participaram do curso a distância profissionais da Atenção Básica de 16 cidades do estado de São Paulo, entre eles: agentes comunitários de saúde, enfermeiros, técnicos de enfermagem, médicos. Foram estabelecidas 25 salas virtuais, com total de 394 participantes. A taxa de evasão desse curso foi estimada em 32,5\%. Esse valor é superior ao apontado pelo Censo $\mathrm{EAD}^{[6]}$, que apontou taxas de evasão entre 11 e 25\%.

A plataforma Moodle foi administrada por um técnico da Universidade/UNESP onde o curso foi oferecido. Todos receberam um link com o endereço da página onde estava hospedada a plataforma, com login e senha de acesso, que ocorreu por computador de mesa ou notebook, com sinal de internet. 
Potencialidades e fragilidades da educação a distância. Russo et al.

A primeira autora deste artigo realizou a análise do conjunto de informações obtidas na plataforma Moodle, composto pelo conteúdo dos fóruns de discussão, das narrativas postadas pelos educandos e das contribuições do tutor na plataforma, assim como, dos registros do planejamento e execução dos projetos de intervenção com a comunidade também disponibilizados em AVA.

O conjunto de dados sistematizados pelo pesquisador foi submetido à técnica da análise de conteúdo, com o intuito de identificar categorias empíricas, com referencial de análise categorial temática de Bardin [7]. Para a discussão das categorias foram utilizados autores do campo da Educação em Saúde em particular e da Saúde Coletiva em geral.

O projeto foi aprovado pelo Comitê de Ética em Pesquisa da FMB/UNESP (protocolo no $372 / 2010$, subprojeto III), e os alunos assinaram um Termo de Consentimento Livre e Esclarecido. Portanto, tiveram a sua identidade preservada, sendo assegurado o anonimato, conforme a Resolução 196/96.

\section{RESULTADOS}

A análise do conjunto de dados resultou na formulação de quatro categorias, sendo elas: 1 - O processo educacional e o educando em ambiente virtual; 2 - O papel do tutor na modalidade de EAD: 3 - Ações intersetoriais e territorialização.

\section{O processo educacional e o educando em ambiente virtual}

A dificuldade com o uso do computador no processo de aculturação com o ambiente virtual foram fragilidades identificadas no processo de análise da participação dos educandos no curso. Essas barreiras identificadas no processo educacional, foram vencidas pelo envolvimento e comprometimento dos educandos, que ao expor suas expectativas, revelaram insegurança, apreensão em relação ao novo e ansiedade, associadas, a grande entusiasmo e animação.

Ao analisar o processo educacional vivenciado pelos educandos foi possível identificar que o curso proporcionou espaço de reflexão, que foi além do ambiente do trabalho e da comunidade. A possibilidade de repensar o seu próprio comportamento alimentar e a motivação para a modificação das práticas familiares, foram interpretadas como um resultado positivo do processo educacional e, portanto, como uma potencialidade do curso-EAD.

A aproximação teoria-prática, vivenciada no processo educacional em AVA, despertou no educando o interesse para construção dos projetos de intervenção, mesmo diante de desafios. Mesmo diante das dificuldades foi possível identificar que o processo educacional permitiu que os educandos aprimorassem a habilidade de releitura da dinâmica de trabalho na atenção primária e na própria comunidade

A apropriação da estrutura da escrita acadêmica, por parte dos educandos imersos na prática do trabalho, mesmo quando se propõem a lançar vôos na busca de novas aprendizagens é uma barreira a ser enfrentada. A carência de elementos que possam promover a habilidade de expressão dos conhecimentos identificados durante o curso, deve ser, portanto, entendido como uma fragilidade a ser enfrentada no processo educacional e não do educando em si.

\section{O papel do tutor na modalidade de EAD}

A participação do tutor na modalidade de EAD foi caracterizada como um orientador, que teve como objetivo motivar os ACS no processo educativo e evitar a desistência. Sua atuação se revelou voltada ao apoio para e elaboração dos exercícios didáticos e do projeto de intervenção. A avaliação pedagógica ocupou lugar de destaque no desenvolvimento do papel dos tutores, identificada na análise do conjunto dos dados.

Os espaços virtuais de diálogo e troca de experiências entre o tutor e os educandos, tais como Fórum, foi uma ferramenta utilizada pelos participantes do Curso. $\mathrm{Na}$ análise dos dados coletados neste espaço foi possível perceber que o mesmo não foi ocupado com toda sua potencialidade pelos participantes do processo educativo. Os tutores, enquanto educadores, ao privilegiar outras ações no processo educacional poderiam ter ampliado mais sua relação dialógica com os educandos e desta maneira 
poderiam ter apoiado mais os educandos para o enfrentamento de suas fragilidades, tal como a dificuldade da produção de textos acadêmicos.

Essas fragilidades identificadas na análise dessa experiência do Curso EAD indicam a necessidade de ampliar o apoio aos educandos, com melhor utilização dos espaços virtuais dialógicos para feed-back dos produtos educacionais em desenvolvimento e para o enfrentamento de lacunas de conhecimento detectadas. Da mesma maneira, compreendemos a necessidade de oferta de melhor formação de tutores de maneira prévia e, igualmente importante, de forma contínua, nos moldes de educação permanente, no decorrer do curso.

\section{Ações intersetoriais e territorialização}

Inseridas nas atividades de elaboração do projeto do curso Interanutri - Modalidade Agente em AVA, os educandos buscaram, em seus locais de origem, elementos para a caracterização da comunidade e seus atores que promoviam ações de SAN no território. A questão do acesso à alimentação no território (em quantidade e qualidade) foi o foco dessa experiência.

Para realizar uma nova leitura da sua comunidade os educandos foram orientados a lançar mão de estratégias de territorialização. Foi configurada como territorialização a delimitação geográfica da comunidade e identificação de aspectos físicos, demográficos, culturais e socioeconômicos de SAN.

A partir da análise dos projetos e dos dados produzidos em AVA, ficou evidente que as comunidades descritas e estudadas pelos educandos, apresentavam características de vulnerabilidade social, tais como: baixa renda, com situações precárias de saneamento básico, segurança pública, transporte público, saúde e lazer. Com relação às questões de Segurança Alimentar foram descritas deficiências de acesso de alimentação com qualidade, tanto do ponto de vista sanitário quanto dos aspectos nutricionais. Além disso, foram percebidas diferenças significativas de conhecimento acerca das suas comunidades.

Os equipamentos sociais identificados pelos educandos, como promotores de ações intersetoriais na comunidade, foram: as escolas, creches, igrejas e pastorais, associação de agricultores, associação de bairros, ONGs e o setor de assistência social municipal e de saúde. Foi evidenciado na análise dos dados, que os setores de Educação Básica e Atenção Primária em Saúde emergiram como protagonistas na prática de intersetorialidade, com ações de educação em saúde.

Movimentos sociais capitaneados por entidades religiosas, presentes nas comunidades, foram apontados nos exercícios de territorialização pelos educandos. A contribuição desses movimentos sociais para a promoção de SAN se configurou com ações tradicionalmente conhecidas, tais como: hortas comunitárias, doação de alimentos, distribuição de cestas básicas e multimistura e oferta de espaço físico para ações de educação em saúde.

Os trabalhos de territorialização revelaram a fragilidade de intersetorialidade para a promoção de SAN nas comunidades. Setores como agricultura, segurança pública, lazer, cultura, transporte, entre outros, não se revelaram como atuantes e integrados no desenvolvimento de ações intersetoriais para $\mathrm{O}$ enfrentamento dos problemas locais que tem relações diretas e indiretas com a SAN da população.

Além da intersetorialidade, o desenvolvimento de atuação interdisciplinar no interior das unidades de saúde foi revelado pela análise dos dados, como estratégias para o enfrentamento dos problemas de alimentação e nutrição nas comunidades, assim como o protagonismo de seus representantes, para alcançar os processos de mudança almejados.

Comunidades de maior vulnerabilidade foram reveladas no processo de análise dos dados, produzidos ao longo do curso em AVA, como cenários das ações mais frequentes de intersetorialidade para promoçãode SAN. Portanto, o exercício de territorialização e intersetorialidade, foi para os educandos uma oportunidade de releitura da comunidade na qual estava inserida sua prática de trabalho, na medida em que as atividades do curso potencializaram a capacidade de aprofundar o conhecimento do contexto das ações de saúde na Atenção Primária. 


\section{DISCUSSÃO}

\section{As vicissitudes do processo ensino- aprendizado em ambiente virtual}

A Metodologia da Problematização destaca-se por encorajar no educando a observação da realidade com olhar crítico. O exercício de associação entre a teoria, trazida pela temática em estudo, com as observações da realidade da comunidade ou da prática do trabalho, despertam no educando percepções de situações que podem ser transformadas.

Proposta por Charlez Maguerez, a Metodologia da Problematização foi denominada por "Método do Arco"[8-9]. Nos processos educativos orientados por essa metodologia, educando e realidade se aproximam e se transformam. Ao educador cabe a ação de motivar no aluno o olhar para a realidade com objetivo de identificar situações que o incomodem. A partir da identificação das situações-problema, pontoschaves são elencados e em seguida realizada a aproximação com a teoria, a formulação de hipóteses para a solução de problemas, levadas para a transformação da realidade (Figura 1).

Figura 1. Arco da Problematização de Charles Maguerez

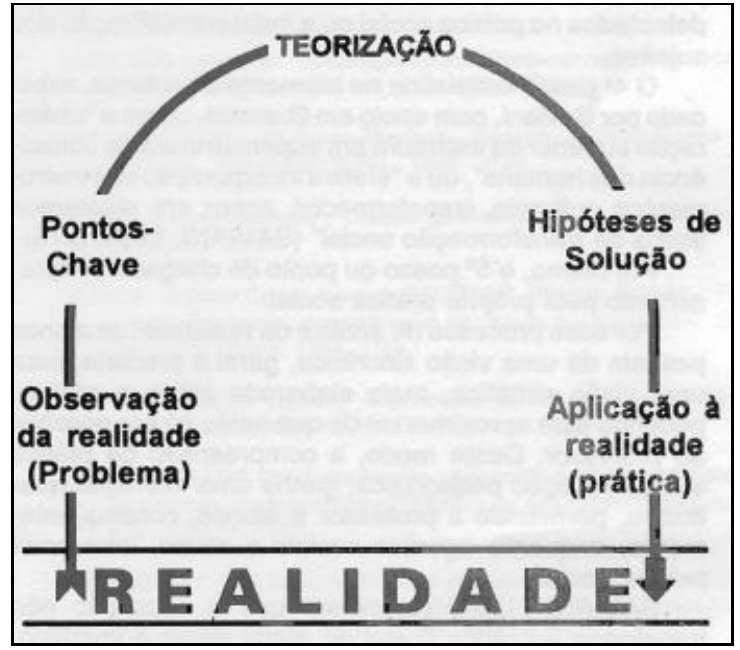

Bastante consolidada a Metodologia da Problematização tem sido também referenciada em estudos que utilizam as tecnologias dos AVA, para os processos de educação a distância [10-11]. Essa estratégia, ao ser utilizada em EAD, mostra-se adequada para o enfrentamento das fragilidades e críticas dessa modalidade de educação, também descrita na literatura ${ }^{[2]}$. Os alunos ao realizarem a leitura da realidade e aproximação da teoria com a prática, são motivados a construir seus projetos de intervenção pautados na educação emancipatória e transformadora ${ }^{[12]}$. Essa escolha metodológica pode trazer ganhos para os cursos de formação em EAD, uma vez que a tecnologia pode atingir número significativo de pessoas em áreas geograficamente distintas ${ }^{[13]}$.

Dessa maneira, uma das principais potencialidades apontadas nos processos de formação EAD é o amplo acesso que o recurso tecnológico permite. Em contrapartida, a utilização da tecnologia pode ser compreendida como fragilidade na medida em que muitos educandos apresentam dificuldades ao lidar com os equipamentos e com os AVA. Essa dificuldade pode gerar medo, ansiedade, apreensão, como visto na análise dos dados desta pesquisa como também apontado na literatura[14].

Autores discutem a importância do ambiente virtual de aprendizagem e seus recursos tecnológicos, como fóruns e chats, para proporcionar aos educandos apoio para elaboração das produções escritas, como uma das etapas do processo de ensinoaprendizagem ${ }^{[2]}$. $\mathrm{O}$ ensino a distância, ao valorizar os espaços e recursos tecnológicos de produção escrita para o enfrentamento das dificuldades com a linguagem acadêmica, pode ser compreendido como um avanço quando comparado com a modalidade presencial de educação tradicional, identificada por Freire como "educação bancária" ou de "transmissão de conhecimento" a qual consegue desenvolver precariamente a capacidade de elaboração textual crítico-reflexiva do educando que "aprende" de forma passiva ${ }^{[15-16]}$.

Desta maneira, a habilidade da escrita dentro dos AVAs formulados a partir da abordagem problematizadora e participativa, pode ser desenvolvida no processo de elaboração dos projetos de intervenção. Contudo, deve-se atentar para a possibilidade de utilização de escritas mais livres que traduzam o processo de construção/interação do educando com sua realidade. Para que isso se viabilize, estratégias de interação com os educandos devem ser consideradas, especialmente ao que diz respeito à mediação dos tutores nos processos de EAD. E, os 
tutores por sua vez, também demandam suporte educacional para o desempenho como educador de ambientes virtuais.

Em revisão de literatura sobre as principais atribuições do tutor no ensino a distância, autores destacam a utilização de recursos lúdicos, conceituaisreflexivos, de atuação com feedback para o desenvolvimento processual e formativo em ambientes virtuais ${ }^{[17]}$. Ou seja, recursos educacionais de concepção participativa-problematizadora utilizados em ambientes presenciais, também podem ser praticados em EAD com resultados animadores, como descritos na literatura ${ }^{[17-18]}$.

Portanto, o processo de ensino-aprendizagem em ambiente virtual tem potencialidades que se associam à formação processual e continuada dos tutores, para promover a mediação da teoria com o campo de vivências, para o aprimoramento do olhar mais crítico sobre o campo de práticas e a prática de territorialização, e, acima de tudo, para não desvalorizar o encontro genuíno e criativo do educador e educando.

\section{A proposta de territorialização no ensino a distância}

A intersetorialidade vem sendo apontada como eixo central de promoção de SAN ${ }^{[19]}$. Contudo, o estabelecimento de inter-relações entre os setores que se associam à garantia da soberania alimentar e da alimentação enquanto direito humano, tem se demonstrado como um desafio a ser superado[20].

Nesse contexto, o conceito de território e territorialização ganha diferentes matizes de significados, de acordo com a aplicação das áreas de conhecimentos que dele se utilizam. $\mathrm{Na}$ saúde, vem sendo compreendido como espaço dotado de singularidades, com delimitações políticoadministrativo e campo de disputas de poderes e conflitos vivenciados no cotidiano, pelos atores sociais, na trama de vínculos institucionais e informais de uma dada realidade ${ }^{[21]}$. A identidade dos espaços físicos é marcada pela sua constituição histórica, com destaque para a concepção de que o território é portador de poder [22]. Essa abordagem é estratégica no fortalecimento do SUS, enquanto política pública, para a consolidação de ações de enfrentamento de problemas e necessidade da população, que habitam e produzem socialmente aquele espaço[23].

A articulação do território, dos diferentes setores e atores sociais revelam-se essenciais também na promoção das ações de segurança alimentar e nutricional, nas delimitações geográficas. Nesse sentido, a aproximação da teoria e da prática em cursos de formação desses atores sociais aponta para a possibilidade de aproximação com as forças do território e percepção de suas potencialidades, assim como, para a identificação de tensões e conflitos, que é fundamental para a execução/implantação de ações de SAN e DHAA[24].

\section{CONCLUSÃO}

Como fragilidades dessa experiência de processo educacional realizado em ambiente virtual, identificamos que as lacunas de conhecimentos ou barreiras no desenvolvimento do processo de ensinoaprendizagem poderiam ter sido melhor superadas com utilização mais adequada das ferramentas do AVA. A interação dialógica em fóruns de discussão permite maior aproximação e vínculo entre educador e educando, para ampliar o aprendizado de ambos.

Em oposição, a aproximação do processo educacional, mesmo em EAD, com o território, compreendido como articulador de forças do movimento social potencializa mudanças nas práticas alimentares e de outras ações no campo da Segurança Alimentar e Nutricional. Cursos ofertados e realizados em ambientes virtuais, ao envolver um número significativo de participantes de comunidades diversas, na mesma temporalidade, se potencializam e, ao mesmo tempo, ampliam as possibilidades de trocas e de construção de redes promotoras de saúde e do DHAA.

\section{REFERÊNCIAS}

[1] Marin MJS, Nascimento EN, Tonhom SFR, Alves SBDA, Otani MAP, Girotto MAet al. Educação permanente: avanços de uma especialização em Saúde da Família na modalidade a distância. RECIIS. 2017;11(4):1-12.

[2] Tavares APC, Leite BS, Silveira IA, Santos TD, Brito WAP, Camacho ACLF. Analysis of Brazilian publications on distance education in nursing: integrative review. Rev. Bras. Enferm. 2018; 71(1): 214-222. 
Potencialidades e fragilidades da educação a distância. Russo $e t$ al.

[3]Brasil. Ministério da Saúde. Secretaria de Atenção à Saúde. Departamento de Atenção Básica. Matriz de ações de alimentação e nutrição na atenção básica de saúde. Série A. Normas e Manuais Técnicos. Brasília (DF): Ministério da Saúde; 2009.

[4] Oliveira MRM, Vieira CM, Galesi, LF. A dimensão presencial de articulação da rede-SANS. In: O tecido da Rede-SANS: histórico, narrativas e reflexões [Internet].2016.São Paulo: CulturaAcadêmica;2016.

[5]Dougiamas M, Taylor P. MOODLE: Using Learning Communities to Create an Open Source Course Management System. Ed-Media [Internet] 2003. [Acesso em 08 out 2018]. Disponível em: http://dougiamas.com/writing/edmedia2003

[6] Associação Brasileira de Educação a Distância (org). Censo EAD. BR: relatório analítico da aprendizagem a distância no Brasil 2016. Curitiba: InterSaberes; 2017.

[7]Bardin L. Análise de conteúdo. 5. ed. Lisboa: Edições 70; 2009.

[8]Bordenave JD, Pereira, AM. Estratégias de ensino aprendizagem. 4.ed. Petrópolis: Vozes, 1982.

[9]Berbel NAN. Metodologia da problematização aplicada a situações de ensino superior. Semina. 1995;16(2):9-19.

[10]Carvalho JS. Educação cidadã a distância: uma perspectiva emancipatória a partir de Paulo Freire [tese].São Paulo: Universidade de São Paulo; 2015.

[11]Amarilla Filho P. Educação a distância: uma abordagem metodológica e didática a partir dos ambientes virtuais. Educação em Revista. 2011;27(2):41-72.

[12] Gadotti M. Perspectivas atuais da educação. São Paulo em Perspectiva. 2000;14(2):03-11.

[13]Cogo ALP. Educação a distância como um espaço de pesquisa para a enfermagem [editorial]. Rev Gaúcha Enferm. 2011;32(2):216.

[14]Sacramento MO. A percepção das emoções na educação a distância: um estudo do processo de ensino e aprendizagem no ambiente online. Ciência (in) Cena Bahia. 2015;(2):17-27.

[15]Freire P. Pedagogia do oprimido. 50a ed. Rio de Janeiro: Paz e Terra; 2011.

[16] Nogueira VS. A linguagem escrita na educação a distância: possibilidades de comunicação e constituição do sujeito/aluno. In: Anais do XV ENDIPE - Encontro Nacional de Didática e Prática de Ensino. Convergências e tensões no campo da formação e do trabalho docente: políticas e práticas educacionais [Internet]; 2010; Belo Horizonte. Belo Horizonte: ENDIPE; 2010.Disponível em: http://w3.ufsm.br/kosmos/textos/vanessa_endipe.pdf
[17]Abreu-e-Lima DM, Alves MN. O feedback e sua importância no processo de tutoria a distância. Pro-Posições. 2011;22(2):189205.

[18] Zeferino A, Domingues R, Amaral E. Feedback como estratégia de aprendizado no ensino médico. Revista Brasileira de Educação Médica. 2007;31(2):176-179.

[19]Burlandy L. Segurança Alimentar e Nutricional: intersetorialidade e as ações de nutrição. Saúde em Revista. 2004;6(13):9-15.

[20]Jaime PC, Delmuè DCC, Campello T, Silva DO, Santos LMP. Um olhar sobre a agenda de alimentação e nutrição nos trinta anos do Sistema Único de Saúde. Ciênc. Saúde coletiva [Internet]. 2018 [acesso em 05 out 2018]; 23(6):1829-1836. Disponível em: http://www.scielo.br/scielo

[21] Ferreira VSC, Silva LMV. Intersetorialidade em saúde: um estudo de caso. In: HartzZMA, Silva LMV. Avaliação em saúde: dos modelos teóricos à prática na avaliação deprogramas e sistemas de saúde. Rio de Janeiro: Editora FIOCRUZ; 2005. p. 103-150.

[22]Raffestin C. Por uma Geografia do Poder. Tradução de Maria Cecília França. São Paulo: Ática; 1993.

[23]Gondim GMM, Monken M, Rojas LI, Barcellos C, Peiter P, Navarro $\mathrm{M}$, et al. O território da saúde: a organização do sistema de saúde e a territorialização. In: Miranda AC, Barcellos C, Moreira JC, Monken M. Território, saúde e ambiente. Rio de Janeiro: Fiocruz; 2008. p.237-255.

[24] Instituto de Economia Agrícola - IEA. Política de Segurança Alimentar eNutricional e a Articulação do Território Brasileiro: questões a debater. Análises e Indicadores do Agronegócio [Internet]. 2011[acesso em 05 out 2018]; 9. Disponível em: http://www.iea.sp.gov.br/ftpiea/AIA/AIA-34-2011.pdf. 